\title{
Is the asymmetry in young infants' categorization of humans versus nonhuman animals based on head, body, or global gestalt information?
}

\author{
PAUL C. QUINN \\ University of Delaware, Newark, Delaware
}

\begin{abstract}
Quinn and Eimas (1998) reported an asymmetry in the exclusivity of the category representations that young infants form for humans and nonhuman animals: category representations for nonhuman animal species were found to exclude humans, whereas a category representation for humans was found to include nonhuman animal species (i.e., cats, horses). The present experiment utilized the familiarization/ novelty-preference procedure with 3 - and 4-month-olds to determine the perceptual cues (i.e., whole stimulus, head alone, body alone) that provided the basis for this asymmetry. The data revealed the asymmetry to be observable only with the whole animal stimuli and not when infants were provided with information from just the head or the body of the exemplars. The results indicate that the incorporation of nonhuman animal species into a category representation for humans is based on holistic information.
\end{abstract}

At some point during development, humans must come to recognize discriminably different objects with important common characteristics as members of the same category. When and how category representations become available have been enduring issues for several generations of investigators, and the results of a number of studies conducted over the last 10 years are consistent with the conclusion that at least some of the abilities needed for category formation are functional in preverbal infants (Quinn, 2002b). In particular, young infants participating in the familiarization/novelty-preference procedure have been shown to form category representations for basiclevel classes chosen from the same superordinate category (Eimas \& Quinn, 1994; Quinn, Eimas, \& Rosenkrantz, 1993). For example, 3- and 4-month-olds familiarized with colored photographic exemplars of domestic cats, representing a variety of breeds, stances, and orientations, generalized their familiarization to novel cats and displayed novel category preferences for birds, dogs, horses, and tigers. In addition, infants familiarized with exemplars of horses generalized their familiarization to novel horses and showed novel category preferences for cats, giraffes, and zebras. The results indicate that the category representation for cats includes novel cats, but excludes birds, dogs, horses, and tigers, and the category

This research was supported by NSF Grant BCS-0096300to PCQ. The author thanks Brian H. Ross and three anonymous reviewers for their comments on an earlier draft of the manuscript. The author also thanks Laurie Yarzab for her assistance in testing participants and Jason Pergola for help with creating the figure. Address correspondence and reprint requests to P. C. Quinn, Department of Psychology, University of Delaware, Newark, DE 19716 (e-mail: pquinn@psych.udel.edu). representation for horses includes novel horses, but excludes cats, zebras, and giraffes. This research suggests that young infants divide the world of objects appropriately into perceptual clusters that later come to have conceptual significance for adults (Quinn \& Eimas, 1997, 2000). ${ }^{1}$

An issue raised by the findings of early categorization concerns the attributes that young infants use to form category representations for nonhuman animal species. Given the static nature of the stimuli and the young age of the infants, the attributes are likely to be perceptual in nature and detectable from the surfaces of the stimuli. In the case of individuated nonhuman animal species such as cats, dogs, horses, and tigers, the diagnostic cues are not obvious inasmuch as all the categories are marked by four legs, eyes, ears, tails, and comparable body shapes.

In a series of experiments, information from a particular part - the head-has been shown to provide a means by which infants form a category representation for cats that excludes dogs (Quinn \& Eimas, 1996; see also Quinn, Eimas, \& Tarr, 2001; Spencer, Quinn, Johnson, \& Karmiloff-Smith, 1997). In one study, 3- and 4-month-olds were randomly assigned to one of three groups: whole animal, head only, or body only. In the whole-animal group, infants were presented with domestic cats and tested with a novel cat paired with a novel dog. The headonly group was presented with the same cats and dogs as was the whole-animal group, but now only their heads were visible; the body information was occluded. The third group, the body-only group, was also familiarized with the same animals shown to the whole-animal group, but in this instance, the head information had been occluded and only the body information was available. The critical finding was that only infants in the whole-animal 
and head-only groups displayed reliable novel category preferences; the body-only group divided their attention between the novel cat and dog bodies. These findings indicate that the head region provides the infants with a sufficient basis to form individuated category representations for cats and dogs. More generally, the data imply that infants categorically represent nonhuman animals on the basis of perceptual part information (see also Rakison \& Butterworth, 1998, for evidence consistent with this suggestion in older infants).

Although the data just described inform us about the skills of young infants in categorizing nonhuman animals, they do not tell us about early abilities to categorize humans. Clearly, any theory of knowledge acquisition about animals would be incomplete without an accounting of how a category representation for humans arises. The data indicating that infants can form category representations for nonhuman animal species suggest that it should be a straightforward matter to demonstrate that infants can form a category representation for humans that excludes exemplars of nonhuman animal species. However, when 3- and 4-month-olds were presented with exemplars of humans depicted in a variety of standing, walking, or running poses and in earth tone (i.e., nonpastel) clothing, the infants formed a category representation for humans that included novel humans, cats, horses, and fish, but excluded cars (Quinn \& Eimas, 1998). This broad representation for humans stands in contrast to the tightly tuned representations for nonhuman animal species that have been found to exclude humans, exemplars from related nonhuman animal species, and cars. There is thus a clear asymmetry with respect to the exclusivity of the representations that young infants use to categorize humans versus nonhuman animal species.

The demonstrated asymmetry in the representations for humans and nonhuman animal species in terms of categorical exclusivity raises the question of whether humans are represented differently from other nonhuman animal species. On the basis of infant performance in preference tests probing the nature of the category representation, Quinn and Eimas (1998) concluded that infants represent humans as individual exemplars belonging to the same category. By contrast, the nonhuman animal species appear to be structured by category-level information, possibly a summary prototype representing an average of the exemplars presented in the laboratory, rather than exemplar-specific details-that is, details about each individual instance of a category (Bomba \& Siqueland, 1983).

How does one explain these differences in the representation of humans and nonhuman animals? Quinn and Eimas (1998) proposed that a broad representation for humans that is based on a wide range of individual exemplars is the result of young infants' considerable familiarity with humans. In effect, over the course of a time span as short as 3-4 months, the differential experience that infants have with humans versus nonhuman animal species has the inductive effect of driving infants to become human "experts." The suggestion is that infants may develop an exemplar-based representation of humans - that is, subordinate-like representations for individual humans clustered together in a representation of humans in general. This broad representation is in opposition to some form of relatively narrow summary representation for other species. For example, the exemplar nature of the human representation could be taken as a cluster of exemplar points defining a human region in psychological space, rather than as a single summary (i.e., prototype) point defining nonhuman animal species (e.g., cats, horses).

An extensive representation for humans may form a special kind of category-a cognitive reference region (Rosch, 1975) - for infants. Because of the size difference between the human region and the cat (or horse) point, the representation for humans would be more accepting of variation along relevant attributes, and thus more likely to incorporate nonhuman animal species. In effect, a category representation for humans could behave as a powerful attractor, somewhat like a perceptual magnet (see, e.g., Kuhl, 1991), that acts to include (i.e., pull in) stimuli with common attributes (e.g., faces, skeletal appendages, elongated bodies). The asymmetry with respect to the categories of nonhuman animals and humans foreshadows Carey's (1985) results showing that 4-year-olds classify a number of nonhuman animals in terms of their similarity to humans. In addition, Pauen (2000), using a habituation-dishabituation procedure, has reported corroborating evidence in 5-month-olds for a broadly inclusive category representation for humans that includes nonhuman animals. Both the infant and the child data suggest that humans may function as an organizing structure for information acquired about other animals.

In the present study, we examined the perceptual information that is the basis for the asymmetry in the category representations for humans versus nonhuman animals among young infants. The experiment followed from the investigation of whether infants form category representations for nonhuman animal species on the basis of information from the head, body, or whole stimulus (Quinn \& Eimas, 1996). Three- and 4-month-olds were familiarized with exemplars of humans or cats and were tested with a novel human versus a novel cat. Within the human and cat conditions, the infants were randomly assigned to one of three experimental groups: whole stimulus, head only, and body only. In the wholestimulus group, infants were familiarized and tested with whole, intact stimuli (head + body). In the head-only group, only the heads of the stimuli were visible; the body information had been occluded. In the body-only group, only the bodies of the stimuli were visible; the head information had been occluded.

If the asymmetry in category representations for humans versus nonhuman animals is governed by the same mechanisms as those for the categorical differentiation of nonhuman animal species from each other (i.e., cats vs. $\operatorname{dog} s$ ), then one would expect the asymmetry to be 
observed in both the whole-stimulus and the head-only conditions, but not in the body-only condition. That is, one would expect the results to parallel the findings obtained in the Quinn and Eimas (1996) study. Alternatively, Quinn and Eimas (1998) suggested that the inclusion of nonhuman animal stimuli into a category representation for humans might be based on the overall structure of the stimuli (i.e., a head adjoined to an elongated body with attached skeletal appendages). If this were the case, one would expect the human versus nonhuman animal asymmetry to be observed in the whole-stimulus condition, but not in the head-only or body-only condition.

\section{METHOD}

\section{Participants}

The participants were 216 infants $3-4$ months of age $(M=99.90$ days; $S D=10.39$ ). One hundred fourteen of the infants were females, and 102 were males. Twenty-eight additional infants were tested; but 16 did not complete the experiment because of fussiness, and 12 were excluded from the data analysis because of failure to compare the test stimuli $(n=5)$, position preference that entailed $95 \%$ or more of the total looking time to one side of the display $(n=5)$, and experimenter error $(n=2)$. The majority of infants were Caucasian and from middle-class homes.

\section{Stimuli}

The stimuli consisted of 18 pictures of cats and 18 pictures of humans. Both stimulus sets were used previously in Quinn and Eimas (1998). Each stimulus picture, of a single cat or a human, was mounted onto a white $17.7 \times 17.7 \mathrm{~cm}$ posterboard for presentation to the infants. Gray construction paper occluded body and head information in the head-only and body-only conditions, respectively. Examples of one human stimulus and one nonhuman animal stimulus in their whole-stimulus, head-only, and body-only depictions are presented in Figure 1.

\section{Apparatus}

The infants were tested in a visual preference apparatus modeled on the one described by Fagan (1970). The apparatus has a display panel with two compartments to hold the stimuli. It was positioned at a distance of $30.5 \mathrm{~cm}$ in front of each infant. The stimuli were illuminated by a fluorescent lamp shielded from the infant's view. A peephole located midway between the two display compartments permitted an observer to record the infant's visual fixations. A second peephole, located directly below the first peephole, permitted a pinhole camera and video recorder to record the infants' gaze durations.

\section{Procedure}

All infants underwent a procedure in which looking times to familiarization and test stimuli were recorded by trained observers who were naive regarding the hypotheses under investigation. Interobserver reliability, as determined by comparing the looking times of 55 infants measured by the observer using the center peephole, and an additional naive observer recording the looking times offline from videotape records, averaged .93 .

Half of the infants were familiarized with 12 cat exemplars and the other half with 12 human exemplars. Within each of these familiar category groups, the infants were randomly assigned to each of three experimental groups: whole stimulus-familiarized with whole stimuli and tested with a novel cat and a novel human; head only-familiarized with heads (body parts occluded) and tested with a novel cat head and a novel human head; body only-familiarized with bodies (heads occluded) and tested with a novel cat body and
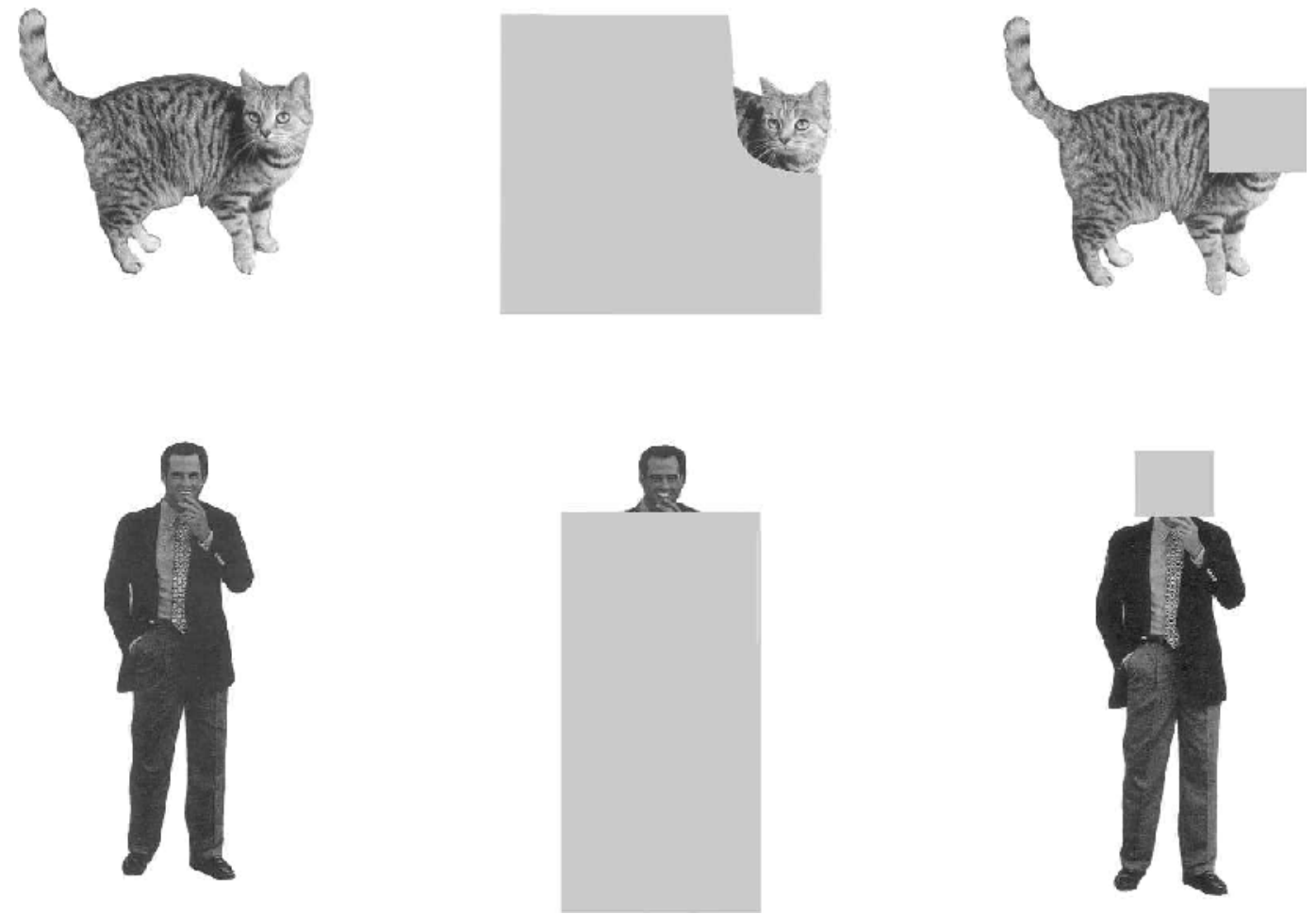

Figure 1. Examples of the nonhuman animal and human exemplars in the whole-stimulus, head-only, and bodyonly depictions. 
a novel human body. During familiarization, each infant was presented with 1 of 36 randomly selected sets of whole stimuli, heads, or bodies. The sets of stimuli were the same across groups, differing only with regard to what parts were presented. Two different wholes, heads, or bodies, were shown on each of six, 15-sec trials, one in the left compartment and one in the right compartment.

The test trials consisted of two 10 -sec trials that paired a novel cat exemplar with a novel human exemplar. There were 36 randomly selected sets of test-trial stimuli, which again were used in all three groups. They differed as before only with respect to the parts that were displayed - whole stimulus, head only, or body only. The leftright positioning of the two stimuli was counterbalanced across infants on the first trial and reversed on the second trial.

\section{RESULTS}

\section{Familiarization Trials}

Individual looking times were summed over the left and right copies of the stimuli on each trial and then averaged across the first and last three trials. Mean fixation times along with their standard deviations are shown in Table 1. An analysis of variance with the factors trial block (1-3 vs. 4-6) $\times$ familiarization category (cat vs. human $) \times$ experimental group (whole stimulus vs. head only vs. body only), performed on the individual looking time scores, revealed a main effect only of trials $[F(2,210)=$ $18.52, p<.001]$. The decrement in looking time across trials provides evidence of a decline in responsiveness with repetitive stimulation that is consistent with the presence of habituation (Cohen \& Gelber, 1975).

\section{Preference Test Trials}

For each infant, a novel category preference score was calculated by dividing the looking time for the novel category stimulus by the looking time for both stimuli during the preference test. Mean novel category preference scores are presented in Table 1. Comparisons of the mean novel category preferences and the chance preference of $50 \%$ were performed in order to identify the experimental group or groups in which the asymmetry in categorization of humans versus nonhuman animals would be observed. In the whole-stimulus group, the mean novel category preference for humans after familiarization with cats was significant, whereas the mean novel category preference for cats after familiarization with humans was not. The asymmetry reported in Quinn and Eimas (1998), a category representation for cats that excludes humans and a category representation for humans that includes cats, was thus replicated. Importantly, however, this asymmetry was not observed in the head-only or body-only groups, irrespective of whether the infants were familiarized with cats or humans. The mean novel category preferences in these groups did not reach significance, even though a substantial number of participants were tested in each cell. Neither head nor body information alone was sufficient to produce the asymmetry.

The same pattern of results was revealed in analyses of the preferences of individual infants. In the wholestimulus group, 29 of 36 infants familiarized with cats and tested with humans had preference scores above the $50 \%$ chance level ( $p=.0001)$, whereas only 21 of 36 infants familiarized with humans and tested with cats had preference scores above the $50 \%$ chance level $(p>.12)$. Moreover, a chi-square test revealed this difference to be reliable $\left[\chi^{2}(1, N=72)=4.18, p<.05\right.$, nondirectional $] .^{2}$ In addition, in the head-only and body-only groups, irrespective of whether the familiar category was cats or humans, the maximum number of infants displaying preference scores above the $50 \%$ chance level was 21 of 36 $(p>.12)$. The asymmetry observed in the mean novel category preference scores-namely, a category representation for cats that excludes humans and a category representation for humans that includes cats-was thus repeated in the analysis of individual preferences. The asymmetry was not observed in the individual preferences of the head-only or body-only groups. Once more, neither head nor body information presented in isolation was sufficient to produce the asymmetry.

\section{DISCUSSION}

The results indicate that the incorporation of nonhuman animal species into a broad category representation for humans by young infants is based on holistic stimulus information. These data are consistent with the suggestion that the category representation for humans is based on the overall structure of the stimuli, such as that of a head region adjoining an elongated body axis with skeletal appendages (Quinn \& Eimas, 1998). The finding that the human representation is based on holistic in-

Table 1

Mean Fixation Times (in Seconds) During the Familiarization Trials and Mean Novel-Category Preference Scores (Percentages) During the Preference Test Trials

\begin{tabular}{|c|c|c|c|c|c|c|c|}
\hline \multirow[b]{3}{*}{ Familiarization Category } & \multicolumn{4}{|c|}{ Fixation Time } & & & \\
\hline & \multicolumn{2}{|c|}{ Trials $1-3$} & \multicolumn{2}{|c|}{ Trials 4-6 } & \multicolumn{3}{|c|}{ Novelty Preference } \\
\hline & $M$ & $S D$ & $M$ & $S D$ & $M$ & $S D$ & $t$ \\
\hline Cat-whole stimulus & 8.46 & 2.15 & 7.72 & 3.12 & 60.07 & 21.50 & $2.81 *$ \\
\hline Cat-head only & 8.47 & 2.43 & 7.92 & 3.18 & 52.47 & 22.77 & 0.65 \\
\hline Cat_-body only & 9.23 & 2.26 & 8.30 & 2.33 & 56.45 & 19.45 & 1.97 \\
\hline Human-whole stimulus & 9.71 & 2.42 & 8.69 & 2.33 & 54.45 & 21.58 & 1.36 \\
\hline Human—head only & 7.98 & 2.44 & 7.59 & 2.93 & 55.66 & 23.21 & 1.64 \\
\hline Human-body only & 9.26 & 2.17 & 7.93 & 2.05 & 56.75 & 23.53 & 1.72 \\
\hline
\end{tabular}

Note- $t$ for mean vs. chance. $* p<.01$, two-tailed. 
formation also contrasts with the findings that representations for nonhuman animal species may be based on part or attribute (i.e., featural) information-such as heads in the case of cats versus dogs (Quinn \& Eimas, 1996).

The data correspond well with the notion that young infants may be representing humans at an "expert" level and with recently proposed ideas regarding the representation of expertise that have emerged in the cognitive neuroscience literature (Gauthier \& Nelson, 2001; Gauthier \& Tarr, 2002). In particular, Gauthier and colleagues have argued that an area of the fusiform gyrus in the brains of adults, once believed to represent faces specifically, actually represents expert knowledge more generally. Moreover, these researchers have demonstrated that expert object recognition by this brain area is characterized by holistic-configural processing. The results reported here are consistent with the idea that young infants may already possess an "expert" representation for humans that is based on holistic information, and imply that "expert" representations may occur early in development with sufficient experience. As neuroimaging techniques are developed for use with infants, it will be interesting to learn whether convergent evidence for this suggestion can be obtained.

The data reported here, when combined with the findings described in the introduction, provide evidence of differences in the ways that young infants represent categories of humans versus nonhuman animals. In particular, young infants categorize nonhuman animals (1) at a basic level of exclusivity, (2) with a summary representation, and (3) on the basis of part information. In contrast, young infants categorize humans (1) at a global level of exclusivity, (2) with an exemplar representation, and (3) on the basis of holistic information. In addition, Quinn (in press) has reviewed evidence indicating that infants categorize nonhuman animals because of learning occurring within the experimental task, whereas infants categorize humans by recruiting from a preexisting knowledge base about humans that arises from experience with humans (see also Quinn, Yahr, Kuhn, Slater, \& Pascalis, 2002).

One may ask on the basis of this evidence whether the differences in the ways that young infants represent humans and nonhuman animals are qualitative or quantitative. One way to think about the differences is that they are quantitative and reflect the effects of experience and the development of expertise. The collection of findings, taken together, lays the foundation for a perceptual learning model of early knowledge acquisition (Quinn, 2002a). Young infants, during the course of early experience, begin to experience numerous objects from various categories. Initially, because of limited experience with a small number of exemplars, the infant's system of representation is likely to consist of individualentities, loosely clustered. However, with sufficient experience, and aided by core abilities to represent within-category sim- ilarity and between-category dissimilarity, the infant may begin to form structured representations organized around summary-level prototypes. The expectation is that such representations (1) would be formed for a variety of generic object categories and (2) could serve as perceptual placeholders for the acquisition of knowledge beyond infancy.

The learning of certain categories may proceed beyond prototypes if infants are provided with additional experience that could come in the form of large numbers of exemplars, or rich or lengthy interaction with a smaller number of exemplars. The summary structure might then become elaborated by the addition of information about individual exemplars. This information serves to both enrich and empower the summary structure, in effect giving rise to the beginnings of an "expert" representation or cognitive reference region. In the infant's overall system of category representation, this region might come to consist of a relatively large number of points in mental space, one point for each exemplar as well as the summary structure. The representational magnification that occurs around these regions of mental space may give rise to psychophysical magnet-like properties.

Early category representations that serve as magnet regions in psychological space may have considerable significance for knowledge acquisition because of their potential to organize large portions of experience. For example, the incorporation of nonhuman animal species into a category representation for humans may be the process that allows for the development of a broad domainlevel category representation for animals in general. From this view, humans may be the "glue" that provides the coherence for a category representation of animals.

As research inquiring into the beginnings of knowledge acquisition continues, one question that remains for the perceptual learning account is to determine more precisely how long-term experiential knowledge for a variety of object classes interacts with short-term experiential knowledge acquired during a series of familiarization trials to produce a particular pattern of looking on preference test trials (Mareschal \& Quinn, 2001). However this question is resolved, the present data have added to our knowledge of early categorization by showing that human infants use holistic information to form a category representation for humans that includes nonhuman animals.

\section{REFERENCES}

Bomba, P. C., \& Siqueland, E. R. (1983). The nature and structure of infant form categories. Journal of Experimental Child Psychology, 35, 294-328.

CAREY, S. (1985). Conceptual change in childhood. Cambridge, MA: MIT Press.

Cohen, L. B., \& Gelber, E. R. (1975). Infant visual memory. In L. B. Cohen \& P. Salapatek (Eds.), Infant perception: From sensation to cognition (Vol. 1, pp. 347-403). New York: Academic Press.

EIMAS, P. D., \& QuINN, P. C. (1994). Studies on the formation of per- 
ceptually based basic-level categories in young infants. Child Development, 65, 903-917.

FAGAN, J. F. (1970). Memory in the infant. Journal of Experimental Child Psychology, 9, 217-226.

Gauthier, I., \& Nelson, C. A. (2001). The development of face expertise. Current Opinion in Neurobiology, 11, 219-224.

GAUTHIER, I., \& TARR, M. J. (2002). Unraveling mechanisms for expert object recognition: Bridging brain activity and behavior. Journal of Experimental Psychology: Human Perception \& Performance, 28, 431-446.

KUHL, P. K. (1991). Human adults and human infants show a "perceptual magnet effect" for the prototypes of speech categories, monkeys do not. Perception \& Psychophysics, 50, 93-107.

MARESCHAL, D., \& QUINN, P. C. (2001). Categorization in infancy. Trends in Cognitive Sciences, 5, 443-450.

PAUEN, S. (2000). Early differentiation within the animate domain: Are humans something special? Journal of Experimental Child Psychology, 75, 134-151.

QUINN, P. C. (2002a). Beyond prototypes: Asymmetries in infant categorization and what they teach us about the mechanisms guiding early knowledge acquisition. In R. V. Kail \& H. W. Reese (Eds.), Advances in child development and behavior (Vol. 29, pp. 161-193). San Diego: Academic Press.

QuINN, P. C. (2002b). Category representation in infants. Current Directions in Psychological Science, 11, 66-70.

QUINN, P. C. (in press). Young infants' categorization of humans versus nonhuman animals: Roles for knowledge access and perceptual process. In L. Gershkoff-Stowe \& D. Rakison (Eds.), Building object categories in developmental time: 32nd Carnegie symposium on cognition (Vol. 32). Mahwah, NJ: Erlbaum.

QuinN, P. C., \& EIMAS, P. D. (1996). Perceptual cues that permit categorical differentiation of animal species by infants. Journal of Experimental Child Psychology, 63, 189-211.

QUINN, P. C., \& EIMAS, P. D. (1997). A reexamination of the perceptualto-conceptual shift in mental representations. Review of General Psychology, 1, 271-287.

QuinN, P. C., \& EIMAs, P. D. (1998). Evidence for a global categorical representation of humans by young infants. Journal of Experimental Child Psychology, 69, 151-174.

QUINN, P. C., \& EIMAS, P. D. (2000). The emergence of category repre- sentations during infancy: Are separate perceptual and conceptual processes required? Journal of Cognition \& Development, 1, 55-61.

QuinN, P. C., EImAS, P. D., \& Rosenkrantz, S. L. (1993). Evidence for representations of perceptually similar natural categories by 3 - and 4month-old infants. Perception, 22, 463-475.

QuinN, P. C., EIMAS, P. D., \& TARR, M. J. (2001). Perceptual categorization of cat and dog silhouettes by 3- to 4-month-old infants. Journal of Experimental Child Psychology, 79, 78-94.

Quinn, P. C., Yahr, J., Kuhn, A., Slater, A. M., \& Pascalis, O. (2002). Representation of the gender of human faces by infants: A preference for female. Perception, 31, 1109-1121.

RAKISON, D. H., \& Butterworth, G. E. (1998). Infants' use of object parts in early categorization. Developmental Psychology, 34, 49-62.

Rosch, E. (1975). Cognitive reference points. Cognitive Psychology, 7, 532-547.

Spencer, J., Quinn, P. C., Johnson, M. H., \& Karmiloff-Smith, A. (1997). Heads you win, tails you lose: Evidence for young infants categorizing mammals by head and facial attributes. Early Development \& Parenting, 6, 113-126.

\section{NOTES}

1. The infants who are presented with exemplars of nonhuman animals (e.g., cats or horses) and form category representations in the laboratory are not necessarily leaving the laboratory with long-term representations for cats and horses that will themselves represent the start-up structures into which subsequent experience and knowledge can be incorporated. The claim is that infants are demonstrating parsing skills in the laboratory that may be successfully deployed to form representations for classes of real objects when those objects are encountered in the natural environment. It is the latter group of representations that may serve as the actual supports for further knowledge acquisition.

2 . The corresponding contrast of mean novel category preference scores was not significant, a null result most likely attributable to the high variability associated with the individual preference scores. Such variability is not unusual for infants performing in infant visual preference tasks.

(Manuscript received August 30, 2002; revision accepted for publication November 26, 2002.) 